PROCEEDINGS OF THE

AMERICAN MATHEMATICAL SOCIETY

Volume 140, Number 8, August 2012, Pages 2913-2927

S 0002-9939(2011)11121-3

Article electronically published on December 1, 2011

\title{
SHARP PHASE TRANSITION THRESHOLDS FOR THE PARIS HARRINGTON RAMSEY NUMBERS FOR A FIXED DIMENSION
}

\author{
ANDREAS WEIERMANN AND WIM VAN HOOF
}

(Communicated by Julia Knight)

\begin{abstract}
This article is concerned with investigations on a phase transition which is related to the (finite) Ramsey theorem and the Paris-Harrington theorem. For a given number-theoretic function $g$, let $R_{c}^{d}(g)(k)$ be the least natural number $R$ such that for all colourings $P$ of the $d$-element subsets of $\{0, \ldots, R-1\}$ with at most $c$ colours there exists a subset $H$ of $\{0, \ldots, R-1\}$ such that $P$ has constant value on all $d$-element subsets of $H$ and such that the cardinality of $H$ is not smaller than $\max \{k, g(\min (H))\}$. If $g$ is a constant function with value $e$, then $R_{c}^{d}(g)(k)$ is equal to the usual Ramsey number $R_{c}^{d}(\max \{e, k\})$; and if $g$ is the identity function, then $R_{c}^{d}(g)(k)$ is the corresponding Paris-Harrington number, which typically is much larger than $R_{c}^{d}(k)$. In this article we give for all $d \geq 2$ a sharp classification of the functions $g$ for which the function $m \mapsto R_{m}^{d}(g)(m)$ grows so quickly that it is no longer provably total in the subsystem of Peano arithmetic, where the induction scheme is restricted to formulas with at most $(d-1)$-quantifiers. Such a quick growth will in particular happen for any function $g$ growing at least as fast as $i \mapsto \varepsilon \cdot \log (\cdots(\log (i) \cdots)$ (where $\varepsilon>0$ is fixed) but not for the function $(d-1)$-times

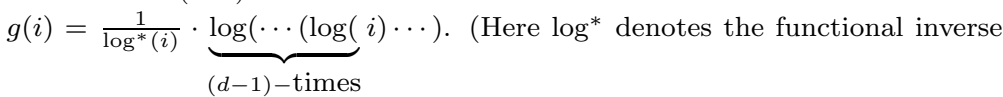
of the tower function.) To obtain such results and even sharper bounds we employ certain suitable transfinite iterations of nonconstructive lower bound functions for Ramsey numbers. Thereby we improve certain results from the article A classification of rapidly growing Ramsey numbers (PAMS 132 (2004), 553-561) of the first author, which were obtained by employing constructive ordinal partitions.
\end{abstract}

\section{INTRODUCTION}

This article is part of a general investigation of phase transitions in logic and combinatorics. The underlying idea is to consider true mathematical assertions $A$ about the natural numbers and to introduce an appropriate order parameter $g$ provided by a number-theoretic function in a meaningful way so that the resulting assertion $A(g)$ is still true for all choices of $g$. In quite a few situations it occurs that, for $g$ of very slow growth, the assertion $A(g)$ follows from a simple set $S$

Received by the editors May 16, 2008 and in revised form, January 22, 2011 and March 4, 2011.

2010 Mathematics Subject Classification. Primary 03F30; Secondary 03D20, 03C62, 05D10.

Key words and phrases. Ramsey theorem, rapidly growing Ramsey functions, fast growing hierarchies, Peano arithmetic.

(C)2011 American Mathematical Society Reverts to public domain 28 years from publication 
of axioms, e.g. (slight extensions of) $I \Delta_{0}+(\exp )$ (formalizing bounded quantifier induction over the natural numbers plus the totality of the exponential function), and that moreover $A(g)$ does not follow from $S$ when $g$ exceeds in growth functions from a certain threshold region. In general we expect that classifying such thresholds is interesting in itself. Moreover, such investigations may shed light on the fundamental question: What makes a given true assertion unprovable from $S$ or even unprovable from stronger systems than $S$ ?

In 9] the first author classified the phase transition emerging from the ParisHarrington $(\mathrm{PH})$ function for unbounded dimensions. That paper also provided upper bounds for the Paris-Harrington numbers of a fixed dimension, but these upper bounds did not match with the lower bounds provided by the Erdös-Rado bounds on the classical Ramsey numbers. The gap for dimension equal to two has been closed in the meantime in [6] via an elementary proof which avoids ordinals completely. In this article we will close the remaining gap for all higher dimensions, and we re-prove the result for dimension two. For this purpose we replace the Ramsey bounds resulting from constructively given ordinal partitions by suitable transfinitely iterated functions for nonconstructive Ramsey bounds refining techniques of Ketonen and Solovay 5 . Intuitively, our proof resembles the behaviour of iterations of a discrete dynamical system at an unstable fixed point (in our case the threshold region). This proof technique is rather far reaching and can be applied (in combination with other techniques) to phase transitions emerging from various combinatorial statements in Ramsey theory. Examples in this regard are provided by the Kanamori-McAloon-Ramsey theorem (regressive Ramsey theorem) [2] and the canonical Ramsey theorem (with largeness condition).

\section{RAmsey functions: Some Basic Results}

In this section we recall some basic terminology and results. We denote the set of natural numbers with $\mathbb{N}$, and we identify a natural number $n \in \mathbb{N}$ with its set of predecessors, so that $n=\{m \in \mathbb{N}: m<n\}$. We also identify $n$ with the half open interval $[0, n[$. In the sequel small Latin letters like $c, d, e, l, m, n, x, y, z$ denote natural numbers. The cardinality of a given set $X$ is denoted by $|X|$, and the set of all $d$-element subsets of a set $X$ is denoted by $[X]^{d}$. For a function $P:[X]^{d} \rightarrow \mathbb{N}$ with $X \subseteq \mathbb{N}$, we write $P\left(x_{0}, \ldots, x_{d-1}\right)$ for $P\left(\left\{x_{0}, \ldots, x_{d-1}\right\}\right)$ under the tacit assumption that $x_{0}<x_{1}<\cdots<x_{d-1}$. We write $X \rightarrow(m)_{c}^{d}$ if for all functions (partitions/colourings) $P:[X]^{d} \rightarrow c$ there exists a set $H \subseteq X$ such that the restriction of $P$ to $[H]^{d}$ is a constant function and $|H| \geq m$. If the restriction of $P$ to $[H]^{d}$ is a constant function, then $H$ is called $P$-homogeneous or $P$-monochromatic.

The classical Ramsey function is then defined as follows:

$$
R_{c}^{d}(m):=\min \left\{R: R \rightarrow(m)_{c}^{d}\right\} .
$$

In this article we investigate a variant of $R_{c}^{d}(m)$ which is defined with respect to the notion of (parameterized) largeness. For a given function $g: \mathbb{N} \rightarrow \mathbb{N}$ we call a nonempty set $H \subseteq \mathbb{N}$ g-large if $|H| \geq g(\min (H))$. We write $X \rightarrow_{g}^{*}(m)_{c}^{d}$ if for all partitions $P:[X]^{d} \rightarrow c$ there exists a $g$-large set $H \subseteq X$ such that the restriction of $P$ to $[H]^{d}$ is a constant function and $|H| \geq m$. The resulting Paris-HarringtonRamsey function is then defined as follows:

$$
R_{c}^{d}(g)(m):=\min \left\{R: R \rightarrow_{g}^{*}(m)_{c}^{d}\right\} .
$$


The following lemmas will be very useful further on. (Let $\log _{2}$ denote the binary $\log$ function with the tacit assumption used througout in this paper that $\log _{2}(0):=$ 0$.

Lemma 2.1. For all $d>0$ there exists a (positive integer) constant $\Gamma>0$ such that $R_{c}^{d+1}(m) \leq 2_{d}\left(\Gamma \cdot c \cdot \log _{2}(c) \cdot m\right)$ for all $c, m>0$.

Proof. See Theorem 26.6 and its proof in [3]. One can take, e.g., $\Gamma:=d ! \cdot 2^{d}$.

Lemma 2.2. There exists a (positive integer) constant $\Lambda$ such that $R_{c}^{2}(m) \geq 2^{\frac{1}{\Lambda} \cdot c \cdot m}$ holds for all natural numbers $c, m$.

Proof. See [1] or [7. One can take $\Lambda:=14$.

Lemma 2.3. For all $d>0$ there exists a (positive integer) constant $\Delta$ such that $R_{c}^{d+1}(m) \geq 2 d\left(\frac{1}{\Delta} \cdot c \cdot m\right)$ for all $c, m$.

Proof. This follows from [3] and Lemma 2.2. In fact we have for $n, c>0$ by Lemma 24.2 of $\left[3\right.$ ] that $n \not \rightarrow(m)_{c}^{r-1}$ implies $2^{n} \not \rightarrow(m+1)_{2 c}^{r}$ for $m \geq r=3$. Moreover, we have for $n, c>0$ by Lemma 24.2 of [3] that $n \not \rightarrow(m)_{c}^{r-1}$ implies $2^{n} \not \rightarrow(2 \cdot m)_{c}^{r}$ for $m \geq r \geq 4$. These bounds together with Lemma 2.2 yield the desired estimate.

Let $\mathrm{PH}_{g}$ denote the assertion

$$
(\forall d, c, m)(\exists R)\left[R \rightarrow_{g}^{*}(m)_{c}^{d}\right],
$$

and let $\mathrm{PH}_{g}^{d}$ denote the assertion

$$
(\forall c, m)(\exists R)\left[R \rightarrow_{g}^{*}(m)_{c}^{d}\right] .
$$

Let $i d$ denote the identity function on $\mathbb{N}$.

Theorem 2.4 (Paris-Harrington Theorem).

(1) The assertion $\mathrm{PH}_{i d}$ is true but not provable from the Peano axioms.

(2) For all $d \geq 2$, the assertion $\mathrm{PH}_{i d}^{d}$ is true but not provable from the fragment of the Peano axioms where the induction scheme is restricted to formulas having at most $(d-1)$-quantifiers.

Proof. This follows, for example, from [5] or [8].

Lemma 2.1 yields that for fixed $d>1$ and a constant function $g$ the assertion

$$
\mathrm{PH}_{g}^{d}
$$

is provable even in $I \Delta_{0}+(\exp )$. So in between constant functions and the identity function there will be a region where there is a transition from provability to unprovability. For first-order Peano Arithmetic (PA), the phase transition threshold for the assertion $\mathrm{PH}_{g}$ has been determined in 9. In this article we determine the corresponding phase transition threshold for the systems of $(d-1)$-quantifier induction and $\mathrm{PH}_{g}^{d}$. To this end we classify the growth rate of the associated function $m \mapsto R_{m}^{d}(g)(m)$ for the relevant number-theoretic functions $g$. The crucial point of this paper is the use of nonconstructive lower bounds from Ramsey theory (cf. Lemma 2.3) which have not been needed in the case of PA. 


\section{Some facts about PARtitions And suitable Sets}

In this section we develop (using ideas and terminology of Ketonen and Solovay 5] ) some basic properties of partitions and suitable sets. We call a partition $P$ : $[\mathbb{N}]^{d} \rightarrow c$ a $(d, c)$-algebra. A partition $P$ is called an algebra if there exist $c, d$ such that $P$ is a $(d, c)$-algebra. For a given function $f: \mathbb{N} \rightarrow \mathbb{N}$, we call a set $S \subseteq \mathbb{N}$ $f$-suitable for a $(d, c)$-algebra $P$ if the following three conditions are satisfied: $S$ is $P$-homogeneous, $|S| \geq d+1$, and $|S| \geq f(\min (S))$.

We say that an algebra $P f$-captures a function $g: \mathbb{N} \rightarrow \mathbb{N}$ if for every $f$-suitable $S$ and all $s, s^{\prime} \in S$ with $s<s^{\prime}$ we have

$$
g(s) \leq s^{\prime}
$$

An algebra $P f$-simulates an algebra $P^{\prime}$ if every $f$-suitable set $S$ for $P$ is also $f$-suitable for $P^{\prime}$.

If $P_{1}:[\mathbb{N}]^{d} \rightarrow c_{1}$ and $P_{2}:[\mathbb{N}]^{d} \rightarrow c_{2}$ are $\left(d, c_{1}\right)$ and $\left(d, c_{2}\right)$ algebras, then we define the product algebra $P:[\mathbb{N}]^{d} \rightarrow c_{1} \cdot c_{2}$ by $P\left(x_{1}, \ldots, x_{d}\right):=c_{1} \cdot P_{1}\left(x_{1}, \ldots, x_{d}\right)+$ $P_{2}\left(x_{1}, \ldots, x_{d}\right)$. Then for a given $f: \mathbb{N} \rightarrow \mathbb{N}, P$ is a $\left(d, c_{1} \cdot c_{2}\right)$-algebra which $f$ simulates $P_{1}$ and $P_{2}$.

Lemma 3.1. Let $f: \mathbb{N} \rightarrow \mathbb{N} c \leq c_{1} \cdot c_{2} \cdot \ldots \cdot c_{k}$. Then any $(d, c)$-algebra can be $f$-simulated by a $\left(d+1, c_{1}+\cdots+c_{k}+1\right)$-algebra.

Proof. Cf. the proof of Corollary 1.2.3 in [5].

Let $\log _{2}^{d}$ be the $d$ th iterate of the binary logarithm function $\log _{2}$ (recall that we put $\left.\log _{2}(0):=0\right)$, and let $m \mapsto 2_{d}(m)$ be the $d$ th iterate of the function $m \mapsto 2^{m}$.

The following lemma is of crucial importance for our further results. The proof of it is already a first example of constructing algebras which capture sufficiently fast growing functions.

Lemma 3.2. Let $d \in \mathbb{N}$ be fixed with $d>0$. For a given $l>0$, let $f_{l}(i):=$ $\max \left\{1,\left\lceil\frac{1}{l} \log _{2}^{d}(i)\right\rceil\right\}$. There exists a constant $c_{l}$ (depending elementarily recursively on l) and $a\left(d+1,\left(c_{l}+1\right) \cdot(d+1)^{2}\right)$-algebra that $f_{l}$-captures the map $m \mapsto 2_{d+1}(m)$.

Proof. We keep $l$ fixed and write $f$ for $f_{l}$. Let

$$
c_{l}=l \cdot \Delta \cdot 2^{(d+1) \cdot 2 \cdot l}
$$

with $\Delta$ from Lemma 2.3. Put $u_{0}:=0, u_{1}:=R_{c_{l}}^{d+1}(d+2)-1$ and for $i>0$

$$
u_{i+1}:=u_{i}+R_{c_{l}}^{d+1}\left(f\left(u_{i}\right)\right)-1 .
$$

We claim that

$$
u_{i} \geq 2_{d}\left(2^{(d+1) \cdot 2 \cdot l \cdot i}\right)
$$

for all $i \geq 1$. Lemma 2.3 yields $u_{1} \geq 2_{d}\left(\frac{1}{\Delta} \cdot c_{l} \cdot(d+2)\right)-1 \geq 2_{d}\left(2^{(d+1) \cdot l \cdot 1}\right)$. Moreover, for the induction step the induction hypothesis yields

$$
\begin{aligned}
u_{i+1} & \geq u_{i}+R_{c_{l}}^{d+1}\left(f\left(u_{i}\right)\right)-1 \geq R_{c_{l}}^{d+1}\left(f\left(u_{i}\right)\right) \\
& \geq 2_{d}\left(\frac{1}{\Delta} \cdot c_{l} \cdot f\left(u_{i}\right)\right) \\
& \geq 2_{d}\left(\frac{1}{\Delta} \cdot c_{l} \cdot \frac{1}{l} \cdot 2^{(d+1) \cdot 2 \cdot l \cdot i}\right)=2_{d}\left(2^{(d+1) \cdot 2 \cdot l \cdot(i+1)}\right) .
\end{aligned}
$$

By the choice of $u_{1}$ we can find a partition $Q_{0}:\left[\left[0, u_{1}[]^{d+1} \rightarrow c_{l}\right.\right.$ such that $|H|<d+2$ for every $Q_{0}$-homogeneous $H$. By choice of $u_{i}$, we can find for $i>0$ a 
partition $Q_{i}:\left[\left[u_{i}, u_{i+1}[]^{d+1} \rightarrow c_{l}\right.\right.$ such that $|H|<f\left(u_{i}\right)$ for every $Q_{i}$-homogeneous $H$. Let us consider the following three algebras:

$$
\begin{aligned}
P_{1}\left(x_{0}, \ldots, x_{d}\right) & = \begin{cases}Q_{i}\left(x_{0}, \ldots, x_{d}\right)+1 & \text { if } u_{i} \leq x_{0}<\cdots<x_{d}<u_{i+1}, \\
0 & \text { otherwise }\end{cases} \\
P_{2}(x, y) & = \begin{cases}0 & \text { if } 2^{x} \leq y, \\
1 & \text { otherwise } \\
0 & \text { if } 2_{d+1}(x) \leq y, \\
1 & \text { otherwise }\end{cases} \\
P_{3}(x, y), & =\text {. }
\end{aligned}
$$

Let $P$ be a $\left(d+1,\left(c_{l}+1\right) \cdot(d+1)^{2}\right)$-algebra which $f$-simulates $P_{1}, P_{2}$, and $P_{3}$ (by first applying Lemma 3.1 to $P_{2}$ and $P_{3}$ and then applying the product algebra construction). Let $S=\left\{x_{0}, \ldots, x_{m-1}\right\}$ be $f$-suitable for $P$. Assume that the restriction of $P_{1}$ to $[S]^{d+1}$ is not equal to 0 . Then there exists an index $i$ such that $S \subseteq\left[u_{i}, u_{i+1}\left[\right.\right.$, and the definition of $Q_{i}$ yields $|S|<d+2$ for $i=0$ or

$$
|S|<f\left(u_{i}\right) \leq f(\min (S))
$$

for $i>0$, contradicting that $S$ is $f$-suitable for $P$. So the restriction of $P_{1}$ to $[S]^{d+1}$ equals the constant function with value 0 . This yields that for any $j$ at most $d$ elements of $S$ are contained in $\left[u_{j}, u_{j+1}\left[\right.\right.$. This means that $\left[u_{0}, u_{j+1}\right.$ [ contains at most $d \cdot(j+1)$ elements of $S$. Thus $x_{m-1} \geq u_{\left\lfloor\frac{m-1}{d}\right\rfloor}$.

We know that $m=|S| \geq f(\min S) \geq \frac{1}{l} \cdot \log _{2}^{d}\left(x_{0}\right)$ and thus

$$
\begin{aligned}
x_{m-1} & \geq u_{\left\lfloor\frac{m-1}{d}\right\rfloor} \geq 2_{d}\left(2^{(d+1) \cdot 2 \cdot l \cdot\left\lfloor\frac{m-1}{d}\right\rfloor}\right) \\
& \geq 2_{d+1}(m \cdot l) \\
& \geq 2_{d+1}\left(\log _{2}^{d}\left(x_{0}\right)\right) \geq 2^{x_{0}},
\end{aligned}
$$

since $d>0$ and $|S|=m \geq d+2$. This yields $P_{2}\left(x_{0}, x_{m-1}\right)=0$; thus

$$
P_{2}\left(x_{0}, x_{1}\right)=P_{2}\left(x_{1}, x_{2}\right)=\cdots=P_{2}\left(x_{m-2}, x_{m-1}\right)=0 .
$$

Since $P$ is $f$-suitable we obtain $m \geq d+2$; hence

$$
x_{m-1} \geq 2^{x_{m-2}} \geq 2^{2^{x_{m-3}}} \geq \cdots \geq 2_{m-1}\left(x_{0}\right) \geq 2_{d+1}\left(x_{0}\right) .
$$

This yields $P_{3}\left(x_{0}, n_{m-1}\right)=0$ and then

$$
P_{3}\left(x_{0}, x_{1}\right)=P_{3}\left(x_{1}, x_{2}\right)=\cdots=P_{3}\left(x_{m-2}, x_{m-1}\right)=0,
$$

proving that $P f$-captures the map $m \mapsto 2_{d+1}(m)$.

The following lemma shows that just by using enough colours in partitions we can guarantee that the minima of suitable sets can be forced to be arbitrarily large.

Lemma 3.3. Let $c, d, e>0$ and $P$ be $a(d, c)$-algebra. Then there exists $a(d, c+e)$ algebra $P^{*} f$-simulating $P$ such that $\min S \geq e$ for every $f$-suitable $S$ for $P^{*}$.

Proof. See Lemma 1.4 in [5]. For convenience we recall the proof. Let $P^{*}:[\mathbb{N}]^{d} \rightarrow$ $e+c$ be defined by $P^{*}(X)=\min (X)$ if $\min (X)<e$ and $P^{*}(X)=e+P(X)$ otherwise. Let $S=\left\{x_{0}, \ldots, x_{m-1}\right\}$ be $f$-suitable for $P^{*}$. This yields $m \geq d+1$. We claim that $x_{0} \geq e$. Otherwise $P^{*}\left(\left\{x_{0}, \ldots, x_{d-1}\right\}\right)=x_{0}$. But then $P^{*}\left(\left\{x_{1}, \ldots, x_{d}\right\}\right)=x_{1}$, contradicting the $f$-suitability of $S$. 


\section{RAPIDLY GROWING FUNCTIONS}

Recall that for any limit ordinal $\lambda<\varepsilon_{0}$ there is a canonical fundamental sequence for $\lambda$ which can be defined recursively as follows:

(1) If $\lambda=\omega^{\alpha+1}(\beta+1)$, then we put $\lambda[x]:=\omega^{\alpha+1} \cdot \beta+\omega^{\alpha} \cdot x$.

(2) If $\lambda=\omega^{\gamma}(\beta+1)$ and $\gamma<\lambda$ is a limit, then we define $\lambda[x]:=\omega^{\gamma} \cdot \beta+\omega^{\gamma[x]}$. For convenience we further put $(\alpha+1)[x]:=\alpha$ and $0[x]:=0$. For each $\alpha<\varepsilon_{0}$, we define the (rapidly growing) function $F_{\alpha}: \mathbb{N} \rightarrow \mathbb{N}$ recursively as follows:

(1) $F_{0}(x)=x+1$.

(2) $F_{\beta+1}(x)=F_{\beta}^{x}(x)$ (where the upper index denotes the number of iterations).

(3) $F_{\lambda}(x)=F_{\lambda[x]}(x)$ if $\lambda$ is a limit.

For a given unbounded weakly increasing function $f: \mathbb{N} \rightarrow \mathbb{N}$, we define a second hierarchy of (typically also rapidly growing) function $B(f)_{\alpha}$ for each $\alpha<\varepsilon_{0}$ as follows:

(1) $B(f)_{0}(x)=2^{x}$.

(2) $B(f)_{\beta+1}(x)=B(f)_{\beta}^{f(x)}(x)$.

(3) $B(f)_{\lambda}(x)=B(f)_{\lambda[f(x)]}(x)$ if $\lambda$ is a limit.

For the specific choices of $f$ in which we are interested, we can establish a connection between the two hierarchies of functions in the following lemma.

Lemma 4.1. Let $d, l>1$ and $f_{l}(i):=\max \left\{1,\left\lceil\frac{1}{l} \cdot \log _{2}^{d}(i)\right\rceil\right\}$. If $x \geq 1$, then

$$
B\left(f_{l}\right)_{\alpha}\left(2_{d}(l \cdot x)\right) \geq 2_{d}\left(l \cdot F_{\alpha}(x)\right) .
$$

Proof. We proceed by induction on $\alpha$. We write $f$ for $f_{l}$ and $B_{\alpha}$ for $B(f)_{\alpha}$. If $\alpha=0$, then $B_{0}(x)=2_{d+1}(l \cdot x) \geq 2_{d}(l \cdot(x+1))=2_{d}\left(l \cdot F_{0}(x)\right)$ since $x \geq 1$ and $l>1$. For $\alpha>0$ the induction hypothesis yields:

$$
\begin{aligned}
B_{\alpha+1}\left(2_{d}(l \cdot x)\right) & =B_{\alpha}^{x-1}\left(B_{\alpha}\left(2_{d}(l \cdot x)\right) \geq B_{\alpha}^{x-1}\left(2_{d}\left(l \cdot F_{\alpha}(x)\right)\right)\right. \\
& =B_{\alpha}^{x-2}\left(B_{\alpha}\left(2_{d}\left(l \cdot F_{\alpha}(x)\right)\right)\right) \\
& \geq B_{\alpha}^{x-2}\left(2_{d}\left(l \cdot F_{\alpha}^{2}(x)\right)\right) \\
& \geq \cdots \geq 2_{d}\left(l \cdot F_{\alpha+1}(x)\right) .
\end{aligned}
$$

If $\alpha$ is a limit ordinal, then the induction hypothesis yields:

$$
\begin{aligned}
B_{\alpha}\left(2_{d}(l \cdot x)\right) & =B_{\alpha\left[\left[\frac{1}{l} \cdot \log _{2}^{d}\left(2_{d}(l \cdot x)\right)\right]\right]}\left(2_{d}(l \cdot x)\right) \\
& \geq B_{\alpha[x]}\left(2_{d}(l \cdot x)\right) \\
& \geq 2_{d}\left(l \cdot F_{\alpha[x]}(x)\right) \\
& =2_{d}\left(l \cdot F_{\alpha}(x)\right) .
\end{aligned}
$$

For a given unbounded weakly increasing function $f$, we define its functional inverse function by

$$
f^{-1}(i):=\min \{j: f(j) \geq i\} .
$$

The following proposition is very useful for further results.

Proposition 4.2. Let $d>0$ and $f$ be a weakly monotone unbounded function, and let $f^{-1}$ be its functional inverse. Then there exists a $\left(d, 1+f^{-1}(2 d+3)\right)$-algebra $G$ such that if $S$ is $f$-suitable for $G$, then $f(\min (S)) \geq 2 d+3$.

Proof. This follows from Lemma 3.3 applied to the trivial algebra $P:[\mathbb{N}]^{d} \rightarrow 1$. Note that the number of colours is here increased by $f^{-1}(2 d+3)$. 
Lemma 4.3. Let $c, e>0$ and $d \geq 0$. Let $h:[\mathbb{N}]^{e} \rightarrow \mathbb{N}$, and assume $h\left(x_{0}, \ldots, x_{e-1}\right)$ $<2_{d}\left(f\left(x_{0}\right)^{c}\right)$, where $f: \mathbb{N} \rightarrow \mathbb{N}$ is a weakly increasing unbounded function. Then there exists an $\left(e+d+1,\left(18+6 \cdot f^{-1}(2 e+3)\right)^{c} \cdot\left(d^{2}+5 d+6\right)^{d}\right)$-algebra $G$ such that for every $f$-suitable $S$ for $G$ there exists a function $g_{S}: S \rightarrow \mathbb{N}$ such that $h(X)=g_{S}(\min X)$ for every $X \in[S]^{e}$.

Proof. This is proved by induction on $d$.

Case 1. $d=0$ and $c=1$.

(1) We define $G_{1}:[\mathbb{N}]^{e} \rightarrow 2$ and $G_{2}:[\mathbb{N}]^{e+1} \rightarrow 3$ and $G_{3}:[\mathbb{N}]^{e+1} \rightarrow 2$ by

$$
\begin{aligned}
G_{1}(X) & = \begin{cases}0 & h(X)<\left\lfloor\frac{f(\min X)}{2}\right\rfloor \\
1 & \text { otherwise }\end{cases} \\
G_{2}\left(x_{0}, \ldots, x_{e}\right) & = \begin{cases}0 & h\left(x_{0}, \ldots, x_{e-1}\right)=h\left(x_{0}, x_{2}, \ldots, x_{n}\right), \\
1 & h\left(x_{0}, \ldots, x_{e-1}\right)>h\left(x_{0}, x_{2}, \ldots, x_{e}\right), \\
2 & h\left(x_{0}, \ldots, x_{e-1}\right)<h\left(x_{0}, x_{2}, \ldots, x_{e}\right) ;\end{cases} \\
G_{3}(X) & = \begin{cases}0 & \text { if } h \text { is min-homogeneous on }[X]^{e}, \\
1 & \text { otherwise. }\end{cases}
\end{aligned}
$$

By Lemmas 3.3 and 3.1 we can find an $\left(e+1,3+\left(f^{-1}(2 e+3)\right)\right)$-algebra $G_{4} f$-simulating $G_{1}$ such that $f(\min (S)) \geq 2 e+3$ for every $f$-suitable $S$ for $G_{1}$. By the product construction we can construct the desired $(e+$ $\left.1,\left(3+f^{-1}(2 d+3)\right) \cdot 2 \cdot 3\right)$-algebra $G$ via $f$-simulating $G_{2}, G_{3}$, and $G_{4}$. Let $S=\left\{x_{0}, \ldots, x_{k}\right\}$ be $f$-suitable for $G$. We show that $G_{4}$ is identical zero on $[S]^{e+1}$. If this is shown, then $h$ depends only on its first coordinate $\min X$.

(2) Define $W=\left\{m: m<\left\lfloor\frac{f\left(x_{0}\right)}{2}\right\rfloor\right\}$ when the restriction of $G_{1}$ to $[S]^{e}$ is zero and $W=\left\{m:\left\lfloor\frac{f\left(x_{0}\right)}{2}\right\rfloor \leq m<f\left(x_{0}\right)\right\}$ otherwise. Then $|W| \leq\left\lfloor\frac{f\left(x_{0}\right)}{2}\right\rfloor+1$. Since $d=0, c=1$ and $h\left(x_{0}, \ldots, x_{e-1}\right)<2_{0}\left(f\left(x_{0}\right)\right)=f\left(x_{0}\right)$, it follows that $h(A) \in W$ if $A \in[S]^{e}$ and $\min A=x_{0}$.

(3) Further in this proof, we shall need the following inequality: $\left\lfloor\frac{f\left(x_{0}\right)}{2}\right\rfloor+1<$ $f\left(x_{0}\right)-e$. Since $f\left(x_{0}\right)-\left\lfloor\frac{f\left(x_{0}\right)}{2}\right\rfloor=\left\lfloor\frac{f\left(x_{0}\right)+1}{2}\right\rfloor$, it suffices to show $e+2 \leq$ $\left\lfloor\frac{f\left(x_{0}\right)+1}{2}\right\rfloor$. But $e+2 \in \mathbb{N}$, so it is enough to show $e+2 \leq \frac{f\left(x_{0}\right)+1}{2}$. Since $S$ is $f$-suitable for $G_{4}$, this implies $f\left(x_{0}\right) \geq 2 e+3$, hence the desired inequality.

(4) For $1 \leq i \leq f\left(x_{0}\right)+1-e$ we define (note that $k+1 \geq f\left(x_{0}\right)$ since $S$ is $f$-large)

$$
A_{i}=\left\{x_{0}, x_{i}, \ldots, x_{i+e-2}\right\},
$$

so $A_{i} \in[S]^{e}$. Further we put $w_{i}:=h\left(A_{i}\right)$, such that $w_{i} \in W$. From (3) and the pigeonhole principle we obtain $i, j$ such that $1 \leq i<j \leq f\left(x_{0}\right)+1-e$ with $w_{i}=w_{j}$.

(5) That $G$ simulates $G_{2}$ yields that $G_{2}$ is constant on $[S]^{e+1}$. Suppose that this value equals 1 ; then $w_{1}>w_{2}>\cdots>w_{f\left(x_{0}\right)+1-e}$, which contradicts (4). For similar reasons, the constant value of $G_{2}$ is not 2 , such that $G_{2}$ is identical 0 on $[S]^{e+1}$.

(6) Since $|S| \geq f(\min S) \geq 2 e+3$, we can talk about $x_{2 e+2}$. We shall prove that $h(Y)=h\left(x_{0}, x_{e+1}, \ldots, x_{2 e-1}\right)$ when $Y \in\left[\left\{x_{0}, \ldots, x_{e}\right\}\right]^{e}$ and $\min Y=x_{0}$. For this we set $\left\{t_{0}, \ldots, x_{2 e-2}\right\}=Y \cup\left\{x_{e+1}, \ldots, x_{2 e-1}\right\}$. Since the restriction 
of $G_{2}$ to $[S]^{e+1}$ is zero, we get:

$$
\begin{aligned}
h(Y) & =h\left(t_{0}, \ldots, t_{e-1}\right)=h\left(t_{0}, t_{2}, \ldots, t_{e}\right)=\cdots=h\left(t_{0}, t_{i}, \ldots, t_{i+e-2}\right) \\
& =\cdots=h\left(t_{0}, t_{e}, \ldots, t_{2 e-2}\right)=h\left(x_{0}, x_{e+1}, \ldots, x_{2 e-1}\right) .
\end{aligned}
$$

(7) From (6) it follows that $h$ is min-homogeneous if the first element is $x_{0}$. But there exists just one $Y \in\left[\left\{x_{0}, \ldots, x_{e}\right\}\right]^{d}$ with the property that $\min Y \neq x_{0}$; hence $h$ is min-homogeneous on $\left[\left\{x_{0}, \ldots, x_{n}\right\}\right]^{d}$, which implies $G_{4}\left(x_{0}, \ldots, x_{e}\right)=0$.

Case 2. $d=0$ and $c>1$.

We can write $h\left(x_{0}, \ldots, x_{e-1}\right)$ in $f\left(x_{0}\right)$-ary notation $h\left(x_{0}, \ldots, x_{e-1}\right)=$ $h_{1}\left(x_{0}, \ldots, x_{e-1}\right) \cdot f\left(x_{0}\right)^{a_{1}}+\cdots+h_{c}\left(x_{0}, \ldots, x_{e-1}\right) \cdot f\left(x_{0}\right)^{a_{c}}$, with $a_{1}<\cdots<a_{c}$ and $h_{i}\left(x_{0}, \ldots, x_{e-1}\right)<f(x)$. For $1 \leq i \leq c$ let $G_{i}^{\prime}$ be a $\left(e+1,18+6 \cdot f^{-1}(2 e+3)\right)$ algebra such that if $S$ is suitable for $G_{i}^{\prime}, h_{i}$ depends only on its first coordinate on $[S]^{e}$. Let $G$ be a $\left(e+1,\left(18+6 \cdot f^{-1}(2 d+3)\right)^{c}\right)$-algebra $f$-simulating $G_{1}^{\prime}, \ldots, G_{c}^{\prime}$. If $S$ is $f$-suitable for $G$, then $h$ depends only on its first coordinate on $[S]^{e}$.

Case 3. $d=d^{\prime}+1$.

Let $Y=\left\{y_{0}, \ldots, y_{e}\right\}$. If $h\left(y_{0}, \ldots, y_{e-1}\right)=h\left(y_{0}, y_{2}, \ldots, y_{e}\right)$, let $g(Y)=0$. Otherwise $g(Y)$ is the largest $j$ at which the binary expansion of the two values of $h$ differ. This yields $g(Y)<2 d_{d^{\prime}}\left(f(\min Y)^{c}\right)$. Let $d^{\prime \prime}:=\left(18+6 \cdot f^{-1}(2 e+3)\right)^{c} \cdot\left(d^{\prime 2}+5 d^{\prime}+6\right)^{d^{\prime}}$ and let $G_{1}$ be the $\left(e+d^{\prime}+1, d^{\prime \prime}\right)$-algebra from the induction hypothesis, applied to $g$, and let $G_{2}$ be a $\left.\left(e+d^{\prime}+1,\left(18+6 \cdot f^{-1}(2 e+3)\right)^{c} \cdot\left(d^{\prime 2}+5 d^{\prime}+6\right)^{d^{\prime}}\right)+f^{-1}(2 e+3)\right)$ algebra simulating $G_{1}$ such that $f(\min S) \geq 2 d+3$ for every $S$ which is $f$-suitable for $G_{1}$. Further we define $G_{3}:[\mathbb{N}]^{e+1} \rightarrow 3$ and $G_{4}:[\mathbb{N}]^{e+1} \rightarrow 2$ by

$$
\begin{gathered}
G_{3}\left(x_{0}, \ldots, x_{e}\right)= \begin{cases}0 & h\left(x_{0}, \ldots, x_{e-1}\right)=h\left(x_{0}, x_{2}, \ldots, x_{e}\right) \\
1 & h\left(x_{0}, \ldots, x_{e-1}\right)>h\left(x_{0}, x_{2}, \ldots, x_{e}\right) \\
2 & h\left(x_{0}, \ldots, x_{e-1}\right)<h\left(x_{0}, x_{2}, \ldots, x_{e}\right)\end{cases} \\
G_{4}(X)= \begin{cases}0 & \text { if } h \text { is min-homogeneous on }[X]^{e} \\
1 & \text { otherwise. }\end{cases}
\end{gathered}
$$

We have

$$
\begin{aligned}
& (3+d) \cdot(2+d) \cdot\left(f^{-1}(2 d+3)+\left(18+6 \cdot f^{-1}(2 e+3)\right)^{c} \cdot\left(d^{2}+5 d^{\prime}+6\right)^{d^{\prime}}\right) \\
& \leq\left(18+6 \cdot f^{-1}(2 e+3)\right)^{c} \cdot\left(d^{2}+5 d+6\right)^{d} .
\end{aligned}
$$

Let $G$ be a $\left.\left(e+d+1,\left(18+6 \cdot f^{-1}(2 e+3)\right)^{c} \cdot\left(d^{2}+5 d+6\right)^{d}\right)\right)$-dimensional algebra $f$-simulating $G_{2}, \ldots, G_{4}$ using Lemma 3.1 and the product construction. Let $S$ be suitable for $G$. We shall show that the restriction of $G_{3}$ to $[S]^{e+1}$ is equal to zero, which will prove the lemma since then the value $\mathrm{f} h(X)$ depends only on $\min (X)$ (following (6) and (7) from the very first case). Aiming at a contradiction, assume that this restriction has value one and let $S=\left\{x_{0}, \ldots, x_{r}\right\}$. Since $G f$-simulates $G_{1}$, it follows $r \geq 2 d+2$. For $1 \leq i \leq 3$, we define $z_{i}:=h\left(x_{0}, n_{i}, \ldots, x_{e-2+i}\right)$. Our assumption yields $z_{1}>z_{2}>z_{3}$. Further we let $j_{0}=g\left(x_{0}, \ldots, x_{d}\right)$ and $j_{1}=$ $g\left(x_{0}, x_{2}, \ldots, x_{d+1}\right)$. Since $G f$-simulates $G_{2}$, there exists a certain $j$ such that $j_{0}=j_{1}=j$.

From the definition of $g$ it follows that the $j$ th digit is the largest binary digit for which $z_{1}$ and $z_{2}$ differ. The inequality $z_{1}>z_{2}$ yields that the $j$ th digit of $z_{2}$ is 
zero, but the other inequality $z_{2}>z_{3}$ yields that the $j$ th digit of $z_{2}$ equals one, a contradiction.

Lemma 4.4. Let $g: \mathbb{N}^{e} \rightarrow \mathbb{N}$ and assume $g\left(x_{0}, \ldots, x_{e-1}\right) \leq f\left(x_{0}\right)$, where $f: \mathbb{N} \rightarrow$ $\mathbb{N}$ is a weakly monotone increasing function. Then there exists a $(e+1,(18+7$. $\left.f^{-1}(2 e+3) \cdot 18\right)$-algebra $G$ such that if $S$ is $f$-suitable for $G$, the following are true:

(1) On $[S]^{e}, g$ depends only on its first coordinate.

(2) If $X, Y \in[S]^{e}$ and $\min X<\min Y$, then $g(X) \leq g(Y)$.

Proof. First we define the (e,2)-algebra $G_{1}$ as follows:

$$
G_{1}(X)= \begin{cases}0 & g(X)<f(\min X) \\ 1 & \text { otherwise }\end{cases}
$$

Further we consider the $\left(e+1,18+6 \cdot f^{-1}(2 e+3)\right)$-algebra $G_{2}$ provided by Lemma4.3 and the $(e, 2)$-algebra $G_{3}$ defined by

$$
G_{3}(X)= \begin{cases}0 & g(X)<\left\lfloor\frac{f(\min X)}{2}\right\rfloor, \\ 1 & \text { otherwise. }\end{cases}
$$

Next we define the $(e+1,2)$-algebra $G_{4}$,

$$
G_{4}\left(x_{0}, \ldots, x_{e}\right)= \begin{cases}0 & g\left(x_{0}, \ldots, x_{e-1}\right) \leq g\left(x_{1}, \ldots, x_{e}\right), \\ 1 & \text { otherwise, }\end{cases}
$$

and the $\left(e+1,18+7 \cdot f^{-1}(2 e+3)\right)$-algebra $G_{5} f$ simulating $G_{2}$ such that for every $f$-suitable $S$ for $G_{2}$ we have $f(\min (S)) \geq 2 e+3$.

Let $G$ be the $\left(e+1,\left(18+7 \cdot f^{-1}(2 e+3)\right) \cdot 3 \cdot 3 \cdot 2\right)$-algebra simulating $G_{1}, G_{3}, G_{4}$, and $G_{5}$ using Lemma 3.1 and the product construction. Let $S$ be an $f$-suitable set for $G$. We shall now show that $g$ satisfies the conditions of the lemma.

Since $G f$-simulates $G_{1}$ and $S$ is $f$-suitable for $G$, we know that $G_{1}$ is constant on $[S]^{e}$. Assume this value is 1 ; then

$$
f(\min (X)) \geq g(X) \geq f(\min (X)),
$$

such that $g(X)=f(\min (X))$. The claim would follow since $f$ is weakly increasing.

From now on we assume that $G_{1}$ equals zero on $[S]^{e}$. Since $G f$-simulates $G_{2}$, the first item of the lemma follows trivially. Let $S=\left\{x_{0}, \ldots, x_{r}\right\}$ and

$$
z_{i}:=g\left(x_{i}, \ldots, x_{i+e-1}\right) \text { for } 1 \leq i \leq r-e+1 .
$$

When $G_{4}$ has (constant) value 0 on $[S]^{e}$, then $z_{0} \leq \cdots \leq z_{r-e+1}$ from which the result follows. To derive a contradiction, assume that $G_{4}$ has constant value 1 which implies $f\left(x_{0}\right)>z_{0}>\cdots>z_{r-e+1}$. Let $W:=\left\{i: i<\left\lfloor\frac{f\left(x_{0}\right)}{2}\right\rfloor\right\}$ when the restriction of $G_{3}$ to $S^{[n]}$ is 0 and $W:=\left\{i:\left\lfloor\frac{f\left(x_{0}\right)}{2}\right\rfloor \leq i<f\left(x_{0}\right)\right\}$ otherwise. Then (by the choice of $G_{1}$ and $\left.G_{3}\right) z_{i} \in W$ for $1 \leq i \leq r-e+1$. Since $i<j$ yields $z_{i}>z_{j}$ we see that $|W| \geq r-e+1$. Since $S$ is $f$-suitable for $G$ we obtain $r+1=|S| \geq f\left(x_{0}\right) \geq 2 e+3$. Hence $|W| \leq\left\lfloor\frac{f\left(x_{0}\right)}{2}\right\rfloor+1<f\left(x_{0}\right)-e \leq r+1-e$, a contradiction. 


\section{Controlling AND CAPturing FUnCtions With Algebras}

In this section we apply the Ketonen-Solovay toolkit for our purposes.

Definition 5.1. Let $\alpha<\beta \leq \varepsilon_{0}$. We write $\beta \rightarrow_{x} \alpha$ if there exists a sequence of ordinals $\gamma_{0}, \ldots, \gamma_{r}$ for which the following is true:

$$
\gamma_{0}=\beta, \gamma_{i+1}=\gamma_{i}[x] \text { for } 0 \leq i<r, \gamma_{r}=\alpha .
$$

Then as usual $\beta \rightarrow_{x} \alpha$ yields $F_{\beta}(x) \geq F_{\alpha}(x)$. Let

$$
T_{d, c, x}=\left\{\alpha: \omega_{d}(c) \rightarrow_{x} \alpha\right\}
$$

with

$$
\omega_{0}(c)=c \text { and } \omega_{k+1}(c)=\omega^{\omega_{k}(c)} .
$$

Definition 5.2. Let $e>0$, and let $g:[\mathbb{N}]^{e} \rightarrow \varepsilon_{0}$. We say that $g$ is weakly $f$ controlled by the algebra $G$ if for every $f$-suitable $S$ for $G, g$ depends only on its first coordinate on $[S]^{e}$.

When $g$ is weakly $f$-controlled by $G$ and $S$ is $f$-suitable for $G$, then we define $g_{S}\left(x_{0}\right)=g\left(x_{0}, \ldots, x_{e-1}\right)$ for any choice of strictly increasing numbers $x_{0}, \ldots, x_{e-1} \in$ $S$.

We say that $g$ is $f$-controlled by $G$ if $g$ is weakly $f$-controlled by $G$ and whenever $S$ is $f$-suitable for $G$ and $x, y \in S$ with $x<y$, then $g_{S}(x) \leq g_{S}(y)$ (if $g_{S}(x)$ and $g_{S}(y)$ are defined).

Lemma 5.3. Let $d \geq 0$, $e \geq 1$, and $g:[\mathbb{N}]^{e} \rightarrow \varepsilon_{0}$, and suppose $g\left(x_{0}, \ldots, x_{e-1}\right) \in$ $T_{d, c, f\left(x_{0}\right)}$ for all strictly increasing sequences $x_{0}<\cdots<x_{e-1}$. Then $g$ is weakly $f$ controlled by a $\left(e+d,\left(d^{2}+5 d+6\right)^{d} \cdot\left(18+7 f^{-1}(2 d+3)^{6 c}\right)\right.$-algebra.

Proof. We may assume $d>0$. By Lemma 4.3 we can choose an $\left(e+d,\left(d^{2}+5 d+6\right)^{d}\right.$. $\left(18+6 f^{-1}(2 e+3)^{6 c}\right)$-algebra (actually a smaller value for $d$ suffices) that insures that $g\left(x_{0}, \ldots, x_{e-1}\right)$ depends only on $x_{0}$ provided $x_{0} \geq 2$, since $\left|T_{d, c, x}\right| \leq 2_{d-1}\left(x^{6 c}\right)$ holds for $x \geq 2$ according to Proposition 2.10 in [5]. Since

$$
\begin{gathered}
\left(d^{2}+5 d+6\right)^{d} \cdot\left(18+6 f^{-1}(2 e+3)^{6 c}\right)+f^{-1}(2) \\
\leq\left(d^{2}+5 d+6\right)^{d} \cdot\left(18+7 f^{-1}(2 e+3)^{6 c}\right),
\end{gathered}
$$

the assertion follows from Lemma 3.3 .

Proposition 5.4. Let $c>0$ and $g:[\mathbb{N}]^{e} \rightarrow \omega^{c}$ and suppose $g\left(x_{0}, \ldots, x_{e-1}\right) \in$ $T_{1, c, f\left(x_{0}\right)}$ for all $x_{0}<\cdots<x_{e-1}$. Then $g$ can be $f$-controlled by a $(e+1,(18+$ $\left.\left.7 f^{-1}(2 e+3) \cdot 18\right)^{c}\right)$-algebra.

Proof. Consider the Cantor normal form of $g$ :

$$
g\left(x_{0}, \ldots, x_{e-1}\right)=\omega^{c-1} \cdot g_{1}\left(x_{0}, \ldots, x_{e-1}\right)+\cdots+\omega^{0} \cdot g_{c}\left(x_{0}, \ldots, x_{e-1}\right) .
$$

Since $g\left(x_{0}, \ldots, x_{e-1}\right) \in T_{1, c, f\left(x_{0}\right)}$, we know for $g_{i}:[\mathbb{N}]^{e} \rightarrow \mathbb{N}$ that $g_{i}\left(x_{0}, \ldots, x_{e-1}\right) \leq$ $f\left(x_{0}\right)$. Lemma 4.4 yields the existence of an $\left(e+1,\left(18+7 f^{-1}(2 e+3)\right) \cdot 18\right)$-algebra $G_{i}$ $f$-controlling $g_{i}$. Let $G$ be the $\left(e+1,\left(18+7 f^{-1}(2 e+3) \cdot 18\right)^{c}\right)$-algebra $f$-simulating $G_{1}, \ldots, G_{c}$. Then $G f$ controls $g$.

If $\lambda$ has the Cantor normal form $\omega^{\alpha_{1}} \cdot x_{1}+\cdots+\omega^{\alpha_{k}} \cdot n_{k}$, we call $\left\{\alpha_{1}, \ldots, \alpha_{k}\right\}$ the support of $\lambda$. If moreover $\lambda^{\prime}$ has the Cantor normal form $\omega^{\beta_{1}} \cdot m_{1}+\cdots+\omega^{\beta_{l}} \cdot m_{l}$ and if $\lambda>\lambda^{\prime}$, then for some $\alpha_{i}$ in the support of $\lambda$ we have that for all $j<i \alpha_{j}=\beta_{j}$ and $m_{j}=n_{j}$ and $\alpha_{i} \geq \beta_{i}$ or $\beta_{i}$ is undefined. Moreover, if $\alpha_{i}=\beta_{i}$, then $n_{i}>m_{i}$.

Let $\left.u_{c, d, e}:=\left(\left(18+7 f^{-1}(2 e+3)\right)^{6 c+1} \cdot\left(d^{2}+5 d+6\right)^{d} \cdot 18 \cdot(d+2)\right)^{d}\right)$. 
Lemma 5.5. Let $d, e \geq 1$. Suppose that $g:[\mathbb{N}]^{e} \rightarrow \varepsilon_{0}$ satisfies $g\left(x_{0}, \ldots, x_{e-1}\right) \in$ $T_{d, c, f\left(x_{0}\right)}$. Then $g$ can be $f$-controlled by a $\left(e+d, u_{c, d, e}\right)$-algebra.

Proof. We proceed by induction on $d$. If $d=1$, then the result follows from Proposition [5.4. So assume $d \geq 2$. From Lemma 5.3 we obtain a $\left(e+d,\left(d^{2}+5 d+\right.\right.$ $\left.6)^{d} \cdot\left(18+7 f^{-1}(2 e+3)^{6 c}\right)\right)$-algebra $G_{0}$ weakly controlling $g$. Further we define the $(e+1,2)$-algebra $G_{1}$ :

$$
G_{1}\left(x_{0}, \ldots, x_{e}\right)= \begin{cases}0 & \text { if } g\left(x_{0}, \ldots, x_{e-1}\right) \leq g\left(x_{1}, \ldots, x_{e}\right) \\ 1 & \text { otherwise. }\end{cases}
$$

We introduce an auxiliary function $h:[\mathbb{N}]^{e+1} \rightarrow \varepsilon_{0}$ as follows:

- When $g\left(x_{0}, \ldots, x_{e-1}\right) \leq g\left(x_{1}, \ldots, x_{e}\right)$, then we set $h\left(x_{0}, \ldots, x_{e}\right)=0$.

- When $g\left(x_{0}, \ldots, x_{e-1}\right)>g\left(x_{1}, \ldots, x_{e}\right)$, then we let $h\left(x_{0}, \ldots, x_{e}\right)$ be equal to the largest element of the support of $g\left(x_{0}, \ldots, x_{e-1}\right)$ at which $g\left(x_{0}, \ldots, x_{e-1}\right)$ is different from $g\left(x_{1}, \ldots, x_{e}\right)$.

For $r:=d-1$ we obtain $h\left(x_{0}, \ldots, x_{e}\right) \in T_{r, c, f\left(x_{0}\right)}$. The induction hypothesis implies the existence of an $\left(e+r, u_{c, r, e}\right)$-algebra $G_{2}$ controlling $h$.

We define a second auxiliary function $h^{\prime}:[\mathbb{N}]^{e+1} \rightarrow \omega$ as follows. When the coefficient of $\omega^{h\left(x_{0}, \ldots, x_{e}\right)}$ in the Cantor normal form of $g\left(x_{0}, \ldots, x_{e-1}\right)$ exists, let $h^{\prime}\left(x_{0}, \ldots, x_{e}\right)$ be equal to this coefficient. If not, let $h^{\prime}\left(x_{0}, \ldots, x_{e}\right)=0$. Since $g\left(x_{0}, \ldots, x_{e-1}\right) \in T_{d, c, f\left(x_{0}\right)}$, we know $h^{\prime}\left(x_{0}, \ldots, x_{e}\right) \leq f\left(x_{0}\right)$. Lemma 4.4 yields an $\left(e+2,\left(18+7 \cdot f^{-1}(2 e+3)\right) \cdot 18\right)$-algebra $G_{3}$ controlling $h^{\prime}$.

We know $e+2 \leq e+d$, but further also $\left(d^{2}+5 d+6\right)^{d} \cdot\left(18+7 f^{-1}(2 e+3)^{6 c}\right)$. $(d+2) \cdot u_{c, r, e} \cdot\left(18+7 \cdot f^{-1}(2 e+3)\right) \cdot 18 \leq u_{c, d, e}$. So let $G$ be the $\left(e+d, u_{c, d, e}\right)-$ product algebra $f$-simulating $G_{0}, \ldots, G_{3}$. We shall now show that $G f$-controls $g$. Let $S=\left\{x_{0}, \ldots, x_{m}\right\}$ be suitable for $G$. Further, we set $S^{\prime}=\left\{x_{i}: i \leq m-e+1\right\}$ and define $g_{S}: S^{\prime} \rightarrow \varepsilon_{0}$ by $g_{S}\left(x_{i}\right)=g\left(x_{i}, \ldots, x_{i+e-1}\right)$. Since $G f$-simulates $G_{0}$, we know $g\left(x_{0}, \ldots, x_{e-1}\right)=g_{S}\left(x_{0}\right)$, for all $x_{0}<\cdots<x_{e-1} \in S$.

Since $G_{1}$ is $f$-simulated by $G, G_{1}$ takes a constant value $v \leq 1$ on $[S]^{e+1}$. When $v$ equals zero, the lemma is proven. Assume that $v=1$. From our assumption we know that there exist $x_{0}, x_{1}$ (with $x_{0}<x_{1}$ ) such that $g_{S}\left(x_{0}\right)>g_{S}\left(x_{1}\right)$. Further, there exists an ordinal $\eta_{S}\left(x_{0}\right)=h\left(x_{0}, \ldots, x_{e}\right) \in \operatorname{support}\left(g_{S}\left(x_{0}\right)\right)$ with coefficient $m_{S}\left(x_{0}\right)=h^{\prime}\left(x_{0}, \ldots, x_{e}\right)$ such that the Cantor normal forms of $g_{S}\left(x_{0}\right)$ and $g_{S}\left(x_{1}\right)$ are equal when the exponents of these forms are bigger than $\eta_{S}\left(x_{0}\right)$, but differ when these exponents are smaller than or equal to $\eta_{S}\left(x_{0}\right) 11$ Since $G f$-simulates $G_{2}$ and $G_{3}$, which $f$-control $h$ and $h^{\prime}$ respectively, the following is true: when $x, y \in S^{\prime}$ with $x<y$, then $\eta_{S}(x) \leq \eta_{S}(y)$ and $m_{S}(x) \leq m_{S}(y)$. Since $m+1 \geq e+2$, we know $\left\{x_{0}, x_{1}, x_{2}\right\} \subseteq S^{\prime} . g_{S}\left(x_{0}\right)$ and $g_{S}\left(x_{1}\right)$ are equal in the part where the exponents are greater than $\eta_{S}\left(x_{0}\right)$, so this is also true for $g_{S}\left(x_{0}\right)$ and $g_{S}\left(x_{2}\right)$. Consequently, $g_{S}\left(x_{1}\right)$ and $g_{S}\left(x_{2}\right)$ are equal in the part where the exponents are greater than $\eta_{S}\left(x_{0}\right)$, i.e. $\eta_{S}\left(x_{1}\right) \leq \eta_{S}\left(x_{0}\right)$. From the previous, we get $\eta_{S}\left(x_{1}\right) \geq \eta_{S}\left(x_{0}\right)$, such that $\eta_{S}\left(x_{1}\right)=\eta_{S}\left(x_{0}\right)$. But this yields $m_{S}\left(x_{1}\right)<m_{S}\left(x_{0}\right)$, a contradiction.

Lemma 5.6. Let $d>1$ and $c>0$ be fixed. For a given $l \in \mathbb{N}$, let $f_{l}(i):=$ $\max \left\{1,\left\lceil\frac{1}{l} \cdot \log _{2}^{d}(i)\right\rceil\right\}$. Then there exists a natural number $t_{l, d, c}$ (which depends elementarily recursively on $l, d, c)$ and $a\left(d+1, t_{l, d, c}\right)$-algebra $f_{l}$-capturing the function $B\left(f_{l}\right)_{\omega_{d-1}(c)}$.

\footnotetext{
${ }^{1}$ The fact that $\eta_{S}\left(x_{0}\right)$ does not depend on $x_{1}$ is a consequence of $G f$-simulating $G_{2}$, which weakly $f$-controls $h$. Since $G_{3}$ is $f$-simulated by $G$, we know that $m_{S}\left(x_{0}\right)$ does not depend on $x_{1}$.
} 
Proof. We abbreviate $f_{l}$ by $f$, and keep $l$ fixed. Define the $(2,2)$-algebra $G_{0}$ as follows:

$$
G_{0}(x, y)= \begin{cases}0 & \text { when } y \geq B(f)_{\omega_{d-1}(c)}(x) \\ 1 & \text { otherwise }\end{cases}
$$

Further, let $G_{1}$ be a $\left(2,2+f^{-1}\left(2_{d}(3 \cdot l)\right)\right)$-algebra simulating $G_{0}$ and such that whenever $S$ is $f$-suitable for $G_{1}, \min (S) \geq 2_{d}(3 \cdot l)$ (according to Lemma 3.3). Next we define $h:[\mathbb{N}]^{2} \rightarrow \varepsilon_{0}$ as follows. When there exists a $\xi \in T_{d-1, c, f(x)}$ such that $B_{\xi}(x) \geq y$, then let $h(x, y)$ be the smallest $\xi$ with this property. Otherwise, we set $h(x, y)=0$. From Lemma 5.5 we know that there exists a $\left(d+1, u_{c, d, 2}\right)$-algebra $G_{2} f$-controlling $h$. Let $c_{l}:=l \cdot \Delta \cdot 2^{(d+1) \cdot 2 \cdot l}$. Lemma 3.2 implies the existence of a $\left(d+1, c_{l}\right)$-algebra $G_{3}$ that $f_{l}$-captures the map $x \mapsto 2_{d+1}(x)$. Finally we define the $(2,3)$-algebra $G_{4}$ as follows:

$$
G_{4}(x, y)= \begin{cases}2 & \text { when } h(x, y) \text { is not a successor, } \\ 0 & \text { when } h(x, y)=\delta+1 \text { and } B(f)_{\delta}^{\lceil f(x)\rceil-1}(x)<y \\ 1 & \text { when } h(x, y)=\delta+1 \text { and } B(f)_{\delta}^{\lceil f(x)\rceil-1}(x) \geq y\end{cases}
$$

Set $t_{l, d, c}:=\left(2+d+f^{-1}\left(2_{d+1}(3 \cdot l)\right)\right) \cdot c_{l} \cdot\left(d+u_{c, d, 2}\right) \cdot(3+d)$ and let $G$ be the $\left(d+1, t_{l, d, c}\right)$-algebra $f$-simulating $G_{0}, \ldots, G_{4}$. We shall prove that $G f$-captures $B(f)_{\omega_{d}(c)}$. Let $S=\left\{x_{0}, \ldots, x_{m}\right\}$ be $f$-suitable for $G$. Since $S$ is $f$-suitable for $G_{0}$, the algebra $G_{0}$ is constant on $[S]^{2}$ with value $v$, say. We shall now show that $v=0$, for suppose otherwise that $v=1$. Let $\{x, y\} \in[S]^{2}$. Since $G_{0}(x, y)=1$, we know $y<B(f)_{\omega_{d-1}(c)}(x)$. We set $\xi_{0}=\omega_{d-1}(c)[f(x)]$, so $y<B_{\xi_{0}}(x)$ and $\xi_{0} \in$ $T_{d-1, c, f(x)}$. Let $\xi_{1}:=h(x, y)$. Then $\xi_{1}$ is the smallest $\alpha$ for which $\alpha \in T_{d-1, c, f(x)}$ and $y \leq B(f)_{\alpha}(x)$ is true. The ordinal $\xi_{1}$ is not a limit ordinal. Assume otherwise. Put $\xi_{2}:=\xi_{1}[f(x)]$. Then $\omega_{d-1}(c) \rightarrow_{f(x)} \xi_{1} \rightarrow_{f(x)} \xi_{2}$ and $\xi_{2}<\xi_{1} \in T_{d-1, c, f(x)}$ and $y \leq B(f)_{\xi_{2}}(x)=B(f)_{\xi_{1}}(x)$. But this contradicts the minimality of $\xi_{1}$. We claim that $\xi_{1}>0$. Assume otherwise that $\xi_{1}=0$. Then $G f$-simulates $G_{3}$ yields

$$
y \geq 2_{d+1}(x)>B(f)_{0}(x),
$$

but this contradicts $y \leq B(f)_{\xi_{1}}(x)=B(f)_{0}(x)$.

So $\xi_{1}=h(x, y)$ has the form $\delta+1$. On $[S]^{2}$, the function $h$ depends only on its first coordinate, say $h(x, y)=\delta(x)+1$ for $x, y \in S$ with $x<y$. When $\{x, y\} \in[S]^{2}$ and $\delta(y)$ is defined, then $\delta(x) \leq \delta(y)$ since $G_{2}$ controls $h$. Since $h(x, y) \rightarrow_{f(x)} \delta(x)$, we know $B(f)_{\delta(x)}(x)<y$. For $0 \leq i<m-1$ we shall now prove that $x_{i+1}>B(f)_{\delta\left(x_{0}\right)}\left(x_{i}\right)$. By the preceding paragraph $x_{i+1}>B(f)_{\delta\left(x_{i}\right)}\left(x_{i}\right)$ and $\delta\left(x_{0}\right) \leq \delta\left(x_{i}\right)$. When $\delta\left(x_{0}\right)=\delta\left(x_{i}\right)$, our claim is trivial. So suppose $\delta\left(x_{0}\right)<\delta\left(x_{i}\right)$. Since $\omega_{d-1}(c) \rightarrow_{f(x)} h(x, y) \rightarrow_{f(x)} \delta(x)$, we get for $x=x_{0}: \omega_{d-1}(c) \rightarrow_{f\left(x_{0}\right)} \delta\left(x_{0}\right)$, and this implies $\omega_{d-1}(c) \rightarrow_{f\left(x_{i}\right)} \delta\left(x_{0}\right)$ (see [5, p. 282]). Further $\omega_{k}(c) \rightarrow_{f\left(x_{i}\right)} \delta\left(x_{i}\right)$ is true, so $\delta\left(x_{i}\right) \rightarrow_{f\left(x_{i}\right)} \delta\left(x_{0}\right)$ (see [5, p. 281]) and then

$$
B(f)_{\delta\left(x_{0}\right)}\left(x_{i}\right) \leq B(f)_{\delta\left(x_{i}\right)}\left(x_{i}\right)<x_{i+1} .
$$

Using iteration we get

$$
x_{i+1}>B(f)_{\delta\left(x_{0}\right)}\left(x_{i}\right)>B(f)_{\delta\left(x_{0}\right)}\left(B(f)_{\delta\left(x_{0}\right)}\left(x_{i-1}\right)\right)>\cdots>B(f)_{\delta\left(x_{0}\right)}^{i+1}\left(x_{0}\right),
$$


which implies $x_{m}>B(f)_{\delta\left(x_{0}\right)}^{m}\left(x_{0}\right)$. The inequality $|S| \geq f(\min S)$ yields $m \geq$ $\left\lceil f\left(x_{0}\right)\right\rceil-1$, hence $x_{m}>B(f)_{\delta\left(x_{0}\right)}^{\left\lceil f\left(x_{0}\right)\right\rceil-1}\left(x_{0}\right)$, and thus $G_{4}\left(x_{0}, x_{m}\right)=0 . S$ is homogeneous for $G_{4}$. Thus $G_{4}\left(x_{0}, x_{1}\right)=G_{4}\left(x_{1}, x_{2}\right)=0$, and so

$$
x_{2}>B(f)_{\delta\left(x_{1}\right)}^{\left\lceil f\left(x_{1}\right)\right\rceil-1}\left(x_{1}\right) \geq B(f)_{\delta\left(x_{0}\right)}^{\left\lceil f\left(x_{0}\right)\right\rceil-1}\left(x_{1}\right) .
$$

$x_{0} \geq 2_{d}(3 \cdot l)$ yields $f\left(x_{0}\right) \geq 3$, and since $G_{4}\left(x_{0}, x_{1}\right)=0$, we obtain

$$
\begin{aligned}
B(f)_{\delta\left(x_{0}\right)}^{\left\lceil f\left(x_{0}\right)\right\rceil-1}\left(x_{1}\right) & \geq B(f)_{\left.\delta\left(x_{0}\right)\right\rceil-1}^{\left\lceil f\left(x_{0}\right)\right.}\left(B(f)_{\delta\left(x_{0}\right)}^{\left\lceil f\left(x_{0}\right)\right\rceil}\left(x_{0}\right)\right) \\
& \geq B(f)_{\delta\left(x_{0}\right)}^{2 \cdot\left\lceil f\left(x_{0}\right)\right\rceil-2}\left(x_{0}\right) \geq B(f)_{\delta\left(x_{0}\right)}^{\left\lceil f\left(x_{0}\right)\right\rceil}\left(x_{0}\right)=B(f)_{\delta\left(x_{0}\right)+1}\left(x_{0}\right) .
\end{aligned}
$$

This implies $x_{2}>B(f)_{\delta\left(x_{0}\right)+1}\left(x_{0}\right)$, but this contradicts $\delta\left(x_{0}\right)+1=h\left(x_{0}, x_{2}\right)$.

\section{RAmsey numbers: Final Result}

Recall that for a given unbounded weakly increasing function $f$, we defined its functional inverse function by

$$
f^{-1}(i):=\min \{j: f(j) \geq i\} .
$$

Moreover, recall that we put $\omega_{0}(c):=c$ and $\omega_{k+1}(c):=\omega^{\omega_{k}(c)}$ and $\omega_{k}:=\omega_{k}(1)$. For $\alpha<\varepsilon_{0}$, let us introduce the following functions $f_{\alpha}$ which play a crucial role for classifying the phase transition:

$$
f_{\alpha}^{d}(i):=\max \left\{1, \frac{\log _{2}^{d}(i)}{F_{\alpha}^{-1}(i)}\right\} .
$$

Theorem 6.1. Let $d>0$ and $\alpha<\varepsilon_{0}$ be fixed. For every $c, k \in \mathbb{N}$ the following is true:

$$
R_{c}^{d+1}(t)+F_{\alpha}(\Gamma \cdot c) \rightarrow_{f_{\alpha}^{d}}^{*}(k)_{c}^{d+1},
$$

with $t:=\max \left(k, F_{\alpha}(\Gamma \cdot c \cdot(\log (c)+1))\right)$ with $\Gamma$ from Lemma 2.1 .

Proof. Let $c, k \in \mathbb{N}$ be arbitrary and set $N:=R_{c}^{d+1}(t)$. Lemma 2.1 yields

$$
\begin{aligned}
N & +F_{\alpha}\left(\Gamma \cdot c \cdot\left(\log _{2}(c)+1\right)\right) \\
& \leq 2_{d}\left(\Gamma \cdot c \cdot \log _{2}(c) \cdot t\right)+F_{\alpha}\left(\Gamma \cdot c \cdot\left(\log _{2}(c)+1\right)\right) \\
& \leq 2_{d}\left(\Gamma \cdot c\left(\log _{2}(c)+1\right) \cdot t\right) .
\end{aligned}
$$

Let $P:\left[\left[0, N+F_{\alpha}\left(\Gamma \cdot c \cdot\left(\log _{2}(c)+1\right)\right)[]^{d+1} \rightarrow c\right.\right.$. Since

$$
N+F_{\alpha}\left(\Gamma \cdot c \cdot\left(\log _{2}(c)+1\right)\right)-F_{\alpha}\left(\Gamma \cdot c \cdot\left(\log _{2}(c)+1\right)\right)=N,
$$

there exists an $H \subseteq\left[F_{\alpha}\left(\Gamma \cdot c \cdot\left(\log _{2}(c)+1\right)\right), N+F_{\alpha}\left(\Gamma \cdot c \cdot\left(\log _{2}(c)+1\right)\right)\right]$ which is homogeneous for $P$ and such that $|H| \geq t$. This implies

$$
\frac{\log _{2}^{d}(\min (H))}{F_{\alpha}^{-1}(\min (H))} \leq \frac{\log _{2}^{d}\left(2_{d}\left(\Gamma \cdot c \cdot\left(\log _{2}(c)+1\right) \cdot t\right)\right)}{F_{\alpha}^{-1}\left(F_{\alpha}\left(\Gamma \cdot c \cdot\left(\log _{2}(c)+1\right)\right)\right)} \leq t \leq|H| .
$$

From this it follows that $H$ is $f_{\alpha}^{d}$-large and that $|H| \geq k$.

Recall that $t_{l, d, c}:=\left(2+d+f^{-1}\left(2_{d+1}(3 \cdot l)\right)\right) \cdot c_{l} \cdot\left(d+u_{c, d, 2}\right) \cdot(3+d)$ using the notation from Lemma 5.6 . 
Theorem 6.2. Let $d>1$ be fixed. Let $t(m):=t_{m, d+1, m}+2_{d}\left(2_{d+1}(m+2) \cdot m\right)$. We have

for all $m \geq 1$.

$$
R_{t(m)}^{d+1}\left(f_{\omega_{d}}^{d}\right)(d+2) \geq F_{\omega_{d}}(m)
$$

Proof. Let $l>0$ be fixed and let $c \geq 0$. Let $t(l, m):=t_{l, d+1, m}+2_{d}\left(2_{d+1}(m+2) \cdot l\right)$ and $f_{l}(i):=\max \left\{1,\left\lceil\frac{1}{l} \cdot \log _{2}^{d}(i)\right\rceil\right\}$ for $i \geq 0$. We claim first that

$$
R_{t(l, m)}^{d+1}\left(f_{l}\right)(d+2) \geq F_{\omega_{d}}(m) .
$$

Assume otherwise. Choose a $\left(d+1, t_{l, d+1, m}\right)$-algebra $G$ which $f_{l}$-captures $B\left(f_{l}\right)_{\omega_{d-1}(m)}$. Choose a $(d+1, t(l, m))$-algebra $G^{*}$ which $f_{l}$-simulates $G$ and such that every $S f$-suitable for $G^{*}$ has $\min (S) \geq 2_{d+1}(m+2)$. By assumption there will exists a $G^{*}$ homogeneous $S \subseteq\left[0, F_{\omega_{d}(m)}\right.$ [ such that $|S| \geq d+2$ and such that $S$ is $f_{l^{-}}$ large. In this situation $S$ is $f_{l}$-suitable for $G^{*}$; hence $\min (S) \geq 2_{d+1}(m+2) . S$ has at least two elements, say $x_{0}<x_{1}$. Lemmas 5.6 and4.1yield $x_{1} \geq B\left(f_{l}\right)_{\omega_{d-1}(m)}\left(x_{0}\right) \geq$ $B\left(f_{l}\right)_{\omega_{d-1}(m)}\left(2_{d+1}(m+2)\right) \geq F_{\omega_{d}}(m)$. This is not possible, and thus the claim above is shown. Now we show for $t(m):=t(m, m)$ that

$$
R_{t(m)}^{d+1}\left(f_{\omega_{d}}\right)(d+2) \geq F_{\omega_{d}}(m) .
$$

Suppose for a contradiction that there exists an $m \geq 1$ for which

$$
R_{t(m)}^{d+1}\left(f_{\omega_{d}}\right)(d+2)<F_{\omega_{d}}(m)
$$

is true. For all $j \leq R_{t(m)}^{d+1}\left(f_{\omega_{d}}(d+2)\right.$, we have $F_{\omega_{d}}^{-1}(j) \leq F_{\omega_{d}}^{-1}\left(F_{\omega_{d}}(m)\right)=m$; hence $\frac{1}{F_{\omega_{d}}^{-1}(j)} \geq \frac{1}{m}$. It is easy to see that in general $f(i) \leq g(i)$ for all $i \leq R_{c}^{d+1}(g)(m)$ yields $R_{c}^{d+1}(f)(m) \leq R_{c}^{d+1}(g)(m)$. So we obtain

$$
\begin{aligned}
& R_{t(m)}^{d+1}\left(f_{\omega_{d}}^{d}\right)(d+2) \\
& \quad \geq R_{t(m)}^{d+1}\left(f_{m}\right)(d+2) \geq F_{\omega_{d}}(m),
\end{aligned}
$$

a contradiction.

Using classical results in proof theory (see, for example, 4] or [10] for a proof), we finally conclude that

$$
m \mapsto R_{m}^{d+1}\left(f_{\alpha}^{d}\right)(d+1)
$$

(hence $\left.m \mapsto R_{m}^{d+1}\left(f_{\alpha}^{d}\right)(m)\right)$ is provable from the axioms of $P A^{-}$plus $d$-quantifier induction iff $\alpha<\omega_{d}$.

\section{REFERENCES}

1. H. L. Abbott. A note on Ramsey's theorem: Canad. Math. Bull. 15 (1972) 9-10. MR0314673 $(47: 3224)$

2. L. Carlucci, G. Lee and A. Weiermann. Sharp thresholds for hypergraph regressive Ramsey numbers Journal of Combinatorial Theory, Series A, 118(2) (2011), 558-585. MR2739504

3. P. Erdős, A. Hajnal, A. Máté and R. Rado. Combinatorial set theory: Partition relations for cardinals. Studies in Logic and the Foundations of Mathematics, 106. North-Holland, 1984. MR7795592 (87g:04002)

4. M. V. Fairtlough and S. S. Wainer. Hierarchies of provably recursive functions, Chapter III in S. Buss (ed.), Handbook of proof theory, Studies in Logic, Vol. 137, Elsevier Science BV (1998), 149-207. MR 1640327(2000a:03063)

5. J. Ketonen and R. Solovay. Rapidly growing Ramsey functions. Annals of Mathematics (2), 113 (1981), 267-314. MR607894 (84c:03100) 
6. M. Kojman, G. Lee, E. Omri and A. Weiermann. Sharp thresholds for the phase transition between primitive recursive and Ackermannian Ramsey numbers. Journal of Combinatorial Theory A 115(6) (2008), 1036-1055. MR 2423347 (2009e:05307)

7. H. Lefmann. A note on Ramsey numbers. Studia Scientiarum Mathematicarum Hungarica 22 (1987), 445-446. MR 932230 (89d:05132)

8. J. B. Paris and L. Harrington. A mathematical incompleteness in Peano arithmetic. In J. Barwise, ed., Handbook of Mathematical Logic, volume 90 of Studies in Logic and the Foundations of Mathematics, pages 1133-1142. North-Holland, 1977. MR0457132 (56:15351)

9. A. Weiermann. A classification of rapidly growing Ramsey functions. Proc. Amer. Math. Soc., 132(2) (2004), 553-561. MR2022381 (2004m:03211)

10. A. Weiermann. Classifying the provably total functions of PA, Bull. Symbolic Logic (12)2 (2006), 177-190. MR2223920 (2006k:03129)

Department of Mathematics, Ghent University, Krijgslane 281 S22, B-9000 Ghent, BELGIUM

E-mail address: Andreas.Weiermann@UGent.be

Department of Mathematics, Ghent University, Krijgslanan 281 S22, B-9000 Ghent, BELGIUM

E-mail address: Wim.Vanhoof@UGent.be 